\title{
Factores de riesgo y de protección relacionados con el consumo de sustancias psicoactivas en estudiantes de enfermería
}

\author{
Blanca Nivia Morales ${ }^{1}$ \\ Merideidy Plazas ${ }^{2}$ \\ Rafael Sanchez $z^{3}$ \\ Carla Aparecida Arena Ventura ${ }^{4}$
}

\begin{abstract}
Este estudio tiene como objetivo identificar la frecuencia de factores de riesgo y de protección relacionados con el consumo de drogas en estudiantes de enfermería. Es un estudio de corte transversal en que se aplicó el instrumento de Factores de Riesgo y de Protección para el Consumo de Sustancias Psicoactivas validado (FRP-SPA) a estudiantes de enfermería. La información se analizó en el programa STATA 10. Se encuestaron 390 estudiantes. Los dominios "preconceptos y valoración", "permisividad social y accesibilidad a SPA", "habilidades sociales y de autocontrol" son factores de riesgo en casi el $100 \%$ de los encuestados para el consumo de drogas. La "Espiritualidad" y "satisfacción con relaciones interpersonales" son los dominios protectores predominantes. Con base en los datos, se puede presumir que los estudiantes no dimensionan los riesgos frente al consumo de alcohol y tabaco, lo que los lleva a considerar su consumo como normal y de aceptación social.
\end{abstract}

Descriptores: Factores de Riesgo; Factores Protectores; Drogas; Sustancias Psicoactivas.

\footnotetext{
1 Enfermera, Magíster en educación de adultos y participación comunitaria. Coordinadora, Facultad de Enfermería, Fundación Universitaria Ciencias de la Salud, Bogotá, Colômbia. E-mail: blanca.n.morales@gmail.com.

${ }^{2}$ Epidemióloga, Coordinadora de pregrado en investigación, Fundación Universitaria Ciencias de la Salud, Bogotá, Colômbia. E-mail: mplazas@fucsalud.edu.co.

${ }_{3}^{3}$ Médico Psiquiatra, Doctor en Salud Mental. E-mail: rfsanches@uol.com.br.

${ }^{4}$ Profesor Doctor, Escola de Enfermagem de Ribeirão Preto, Universidade de São Paulo, Centro Colaborador de la OMS para el Desarrollo de la Investigación en Enfermería, SP, Brasil. E-mail: caaventura@eerp.usp.br.
}

Correspondencia:

Carla Aparecida Arena Ventura

Universidade de São Paulo. Escola de Enfermagem de Ribeirão Preto

Departamento de Enfermagem Psiquiátrica e Ciências Humanas

Av. dos Bandeirantes, 3900

Bairro: Monte Alegre

CEP: 14040-902 Ribeirão Preto, SP, Brasil

E-mail: caaventura@eerp.usp.br 


\section{Fatores de risco e proteção relacionados ao consumo de substâncias psicoativas em alunos de graduação em enfermagem}

O estudo apresentou como objetivo identificar a frequência de fatores de risco e proteção, relacionados ao consumo de drogas, em estudantes de enfermagem. É estudo de coorte transversal, onde foi aplicado instrumento de fatores de risco e proteção para o consumo de substâncias psicoativas validado (FRP-SPA) para alunos de graduação em enfermagem. Os dados foram analisados por meio do programa STATA 10. Participaram do estudo 390 estudantes. Os domínios "pré-conceito e valoração", "permissividade social e acesso a SPA", "habilidades sociais e autocontrole" são fatores de risco para o consumo de drogas em quase $100 \%$ dos participantes. "Espiritualidade" e "satisfação com as relações interpessoais" são domínios protetores predominantes. Com base nos dados, pode-se presumir que os estudantes não dimensionam os riscos frente ao consumo de álcool e cigarro, o que os leva a considerar seu consumo como normal e de aceitação social.

Descritores: Fatores de Risco; Fatores Protetores; Drogas; Substâncias Psicoativas.

\section{Risk and Protection Factors Related to the Consumption of Psychoactive Substances in Undergraduate Nursing Students}

This study aims to identify the frequency of risk and protection factors related to drug consumption among undergraduate nursing students. It is a cross-sectional study in which authors applied the instrument Risk and Protection Factors for the Consumption of Psychoactive Substances, validated for use with undergraduate nursing students. Data were analyzed through STATA 10. Three hundred and ninety students participated in the study. The domain "prejudice and appraisal", "social permissiveness and access to psychoactive substances", "social skills and self-control" are risk factors for drugs use in $100 \%$ of participants. "Spirituality" and "satisfaction with interpersonal relations" were predominant protective domains. Based on data, authors can conclude that the students did not consider the risks in alcohol and tobacco consumption, as they think it is normal and socially acceptable.

Descriptors: Risk Factors; Protection Factors; Drugs; Psychoactive Substances.

\section{Introducción}

Aunque el consumo de las sustancias psicoactivas (SPA) ha sido un fenómeno universal y constante en la historia de la humanidad, hoy plantea desafíos producto de factores como la globalización, la tecnificación y la comunicación que han desplazado el uso tradicional de sustancias, regulado con clara significación cultural e imponiendo nuevos patrones de consumo caracterizados por sustancias y métodos cada vez más accesibles y potentes ${ }^{(1-3)}$. Las SPA son todas las sustancias de origen natural (sin prescripción médica) con la intención de alterar la percepción, la conciencia o cualquier otro estado psicológico, incluyendo sustancias lícitas o ilícitas, que cumplen con los criterios de abuso y/o dependencia (potencial adictivo, de desarrollo de tolerancia y de síndrome de abstinencia) descritos en los Manuales de Psiquiatría como el DSM IV(4). El estudio Nacional de Salud Mental realizado en el año 2003, en Colombia, muestra que la 
prevalencia de vida de trastornos por sustancias es de $10,6 \%$. El abuso de alcohol es el principal problema. La prevalencia de vida para el alcohol es de $6.7 \%$, lo que indica que uno de cada 15 colombianos abusa del alcohol. La diferencia por sexo es marcada, si se tiene en cuenta que uno de cada 10 hombres abusa del alcohol

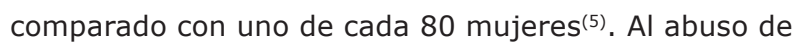
alcohol le sigue en importancia la dependencia a esta sustancia (alcoholismo) con una prevalencia de 2,3\%. Le siguen después la dependencia a la nicotina con una prevalencia de vida de $1,6 \%$, siendo seis veces mayor en hombres que en mujeres. Por otro lado, una de cada 100 personas abusa de sustancias y aproximadamente una de cada 200 tiene dependencia a éstas. Cuando se analiza el uso de drogas, la de mayor prevalencia de vida es la marihuana con un $11,1 \%$, seguida de la cocaína con $3,7 \%$ y tranquilizantes $2,6 \%{ }^{(5)}$.

Las tasas de experimentación de una sustancia están muy relacionadas con factores como el ambiental que se asocia con la disponibilidad y accesibilidad(6-7). Otros factores se relacionan con las motivaciones individuales como la curiosidad, necesidad de evasión a situaciones, necesidad de sentirse aceptado por el grupo, búsqueda de placer, rebeldía y sentido del riesgo, la carencia de objetivos, ilusiones y aspiraciones en la

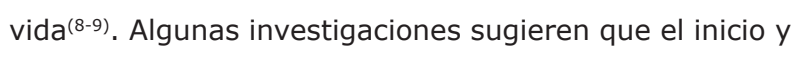
la experimentación con una sustancia se explican mejor por factores externos, mientras que la permanencia en el consumo hasta alcanzar criterios para dependencia se relaciona más con vulnerabilidad determinada por factores genéticos ${ }^{(8-10)}$. Los factores mencionados anteriormente son algunos de los más conocidos, que al coaccionar en forma dinámica propenden a que los jóvenes usen y abusen de las SPA; por tanto, el uso de estas sustancias no es unicausal, el consumo procede de la interacción de diferentes factores en conjunto con las condiciones de oferta; también hay que tener en cuenta los factores protectores que se relacionan con las características individuales y los ambientes socio familiares inmediatos jugando un papel importante frente al consumo(10-12).

Se entienden como factores de riesgo y/o protectores una serie de circunstancias o eventos de naturaleza biológica, psicológica, social o ambiental, cuya presencia o ausencia modifican la probabilidad de la aparición de un problema. La fuerza o importancia de un factor puede cambiar de un grupo a otro o de un individuo a otro, su interacción es dinámica e intrincada, por lo cual su importancia también es variable y no fácil de comprender ${ }^{(12)}$. Ante la complejidad de la problemática, los estudios y la vigilancia del fenómeno adquieren especial relevancia permitiendo evaluar políticas, elaborar programas adecuados y efectivos para la prevención, diseñando intervenciones tempranas para los casos identificados ${ }^{(12-13)}$. Es así como la Comisión Interamericana para el Control del Abuso de Drogas (CICAD) de la Organización de los Estados Americanos, desde los años 80, promueve la formación de recursos humanos especializados para trabajar con el fenómeno de las drogas en las Américas, en las áreas de reducción de la demanda y de la oferta, desarrollo institucional, desarrollo alternativo, lavado de dinero, desarrollo legal, policial y observatorio de drogas ${ }^{(8-13)}$ En este contexto, el presente trabajo se enmarca dentro de la Política Nacional para la Reducción del Consumo de Sustancias Psicoactivas y su impacto para Colombia y buscó identificar los factores de riesgo y de protección relacionados con el consumo de SPA en estudiantes de una facultad de enfermería(14).

\section{Metodología}

Se realizó un estudio de corte transversal en todos los estudiantes de una Facultad de Enfermería que estaban activos en el segundo semestre de 2008 y que desearon participar en el estudio. Fue utilizado un cuestionario de factores de riesgo y de protección para el consumo de drogas en jóvenes(13-14), conformado por 53 ítems que evalúan la presencia/ausencia de seis factores agrupados en 6 dominios: emocional, satisfacción con relaciones interpersonales, preconceptos y valoración de las SPA, espiritualidad, permisividad social $y$ accesibilidad a las SPA y habilidades sociales y de autocontrol dando cuenta del riesgo y protección para el consumo de SPA. El factor emocional se puntúa de 0 a 4 como Protector (FP) y de 5 a 13 como Factor de Riesgo (FR); el dominio satisfacción con relaciones interpersonales tiene un puntaje de 0 a 4 (FR) y de 5 a 10 (FP); preconceptos y valoración de las SPA tiene FR de 0 a 7 y FP de 8 a 28, espiritualidad tiene FR de 0 a 2 y FP de 3 a 6, permisividad social y accesibilidad a las SPA tiene FR de 0 a 2 y FP de 3 a 8 y habilidades sociales y de autocontrol tiene FR de 0 a 3 y FP de 4 a 7. En los ítems positivos, el puntaje para la respuesta afirmativa fue 0 y para la respuesta negativa 1 ; en los ítems negativos se puntúo al revés ${ }^{(15-16)}$.

Además se incluyeron las siguientes variables: estado civil, edad, estrato socioeconómico, sexo, dependencia económica, procedencia y personas con quien vive. Estas variables se analizaron con medidas 
de tendencia central, dispersión y distribución de frecuencias. Los datos fueron analizados en STATA 10.

El estudio fue aprobado por el Comité de Ética e Investigación de la $\operatorname{FUCS}^{(17)}$ y los sujetos de la investigación firmaron el Término de Consentimiento Libre y Esclarecido después de concordaren en participar del estudio.

\section{Resultados}

Se encuestaron 390 estudiantes quienes contestaron de manera voluntaria y firmaron el Término de Consentimiento Libre y Esclarecido; la edad promedio fue de 20 años (DE: 2,7 años). La mayoría de los estudiantes se encontraban entre I y IV semestre $(61,5 \%)$; el sexo que predominó fue el femenino (357), donde el $92,1 \%$ eran solteras (327). El 63,3\% de los estudiantes dependen económicamente de los dos padres, mientras el $24,4 \%$ depende de alguno de los dos, lo que se relaciona directamente con quien viven los estudiantes. El porcentaje restante (12,3\%) manifiesta vivir con algún familiar. El $87,9 \%$ de los estudiantes procede del área urbana.

En la Tabla 1 se puede apreciar que en los primeros semestres hay más estudiantes en riesgo en cada uno de los factores. De esta forma, los preconceptos y la valoración de las SPA, la permisividad social y accesibilidad a las SPA son los más destacados.

Tabla 1 - Factores de Riesgo y de protección para el uso de drogas en los primeros semestres de la carrera de enfermería

\begin{tabular}{|c|c|c|c|c|c|c|}
\hline \multirow{3}{*}{ Semestre } & \multicolumn{2}{|c|}{ Malestar emocional } & \multicolumn{2}{|c|}{$\begin{array}{c}\text { Satisfacción con relaciones } \\
\text { interpersonales }\end{array}$} & \multicolumn{2}{|c|}{$\begin{array}{l}\text { Preconceptos y valoración de } \\
\text { las SPA }\end{array}$} \\
\hline & Protección & Riesgo & Protección & Riesgo & Protección & Riesgo \\
\hline & $\mathrm{N}(\%)$ & $\mathrm{N}(\%)$ & $\mathrm{N}(\%)$ & $\mathrm{N}(\%)$ & $\mathrm{N}(\%)$ & $\mathrm{N}(\%)$ \\
\hline 1 & $26(44,1)$ & $33(55,9)$ & $60(100)$ & 0 & 0 & $35(100)$ \\
\hline II & $35(51,5)$ & $33(48,5)$ & $67(97,1)$ & $2(2,9)$ & $1(1,9)$ & $52(98,1)$ \\
\hline III & $32(66,7)$ & $16(33,3)$ & $47(97,9)$ & $1(2,1)$ & $1(2,5)$ & $39(97,5)$ \\
\hline IV & $27(52,9)$ & $24(47,1)$ & $47(94,0)$ & $3(6,0)$ & 0 & $33(100)$ \\
\hline V & $18(72)$ & $7(28)$ & $25(100)$ & 0 & 0 & $21(100)$ \\
\hline VI & $22(55)$ & $18(45)$ & $43(100)$ & 0 & 0 & $35(100)$ \\
\hline VII & $23(63,9)$ & $13(36,1)$ & $38(100)$ & 0 & 0 & $20(100)$ \\
\hline VIII & $24(82,8)$ & $5(17,2)$ & $30(100)$ & 0 & 0 & $25(100)$ \\
\hline NR & $2(66,7)$ & $1(33,3)$ & $2(66,7)$ & $1(33,3)$ & 0 & $2(100)$ \\
\hline \multirow[t]{2}{*}{ Total } & $209(58,2)$ & $150(41,8)$ & $359(98,1)$ & $7(1,9)$ & $2(0,8)$ & $262(99,2)$ \\
\hline & \multicolumn{2}{|c|}{ Espiritualidad } & \multicolumn{2}{|c|}{$\begin{array}{c}\text { Permisividad social y accesibilidad a } \\
\text { las SPA }\end{array}$} & \multicolumn{2}{|c|}{$\begin{array}{l}\text { Habilidades sociales y de } \\
\text { autocontrol }\end{array}$} \\
\hline \multirow[t]{2}{*}{ Semestre } & Protección & Riesgo & Protección & Riesgo & Protección & Riesgo \\
\hline & $\mathrm{N}(\%)$ & $\mathrm{N}(\%)$ & N (\%) & $N(\%)$ & $N(\%)$ & $N(\%)$ \\
\hline 1 & $45(78,9)$ & $12(21,1)$ & $18(33,3)$ & $36(66,7)$ & $14(23,7)$ & $45(76,3)$ \\
\hline ॥ & $57(85,1)$ & $10(14,9)$ & $20(28,6)$ & $50(71,4)$ & $29(41,4)$ & $41(58,6)$ \\
\hline III & $33(70,2)$ & $14(29,8)$ & $19(40,4)$ & $28(59,6)$ & $22(45,8)$ & $26(54,2)$ \\
\hline IV & $42(82,4)$ & $9(17,6)$ & $17(32,7)$ & $35(67,3)$ & $22(42,3)$ & $30(57,7)$ \\
\hline v & $24(88,9)$ & $3(11,1)$ & $7(26,9)$ & $19(73,1)$ & $7(26,9)$ & $19(73,1)$ \\
\hline $\mathrm{VI}$ & $33(76,7)$ & $10(23,3)$ & $9(22,0)$ & $32(78,0)$ & $12(27,9)$ & $31(72,1)$ \\
\hline VII & $26(72,2)$ & $10(27,8)$ & $9(25,0)$ & $27(75,0)$ & $13(35,1)$ & $24(64,9)$ \\
\hline VIII & $26(81,3)$ & $6(18,8)$ & $4(12,5)$ & $28(87,5)$ & $17(53,1)$ & $15(46,9)$ \\
\hline$N R$ & $2(66,7)$ & $1(33,3)$ & $2(66,7)$ & $1(33,3)$ & $2(66,79)$ & $1(33,3)$ \\
\hline Total & $288(79,3)$ & $75(20,7)$ & $105(29,1)$ & $256(70,9)$ & $138(37,3)$ & $232(62,7)$ \\
\hline
\end{tabular}

Entre los 16 y 20 años fue más frecuente encontrar los factores preconceptos y valoración de las SPA, habilidades sociales y de autocontrol, permisividad social y accesibilidad para las SPA como riesgo para el consumo de SPA (Tabla 2). 
Tabla 2 - Factores de Riesgo y de protección para el uso de drogas según grupos de edad de los estudiantes

\begin{tabular}{|c|c|c|c|c|c|c|}
\hline \multirow{3}{*}{ Grupos de edad } & \multicolumn{2}{|c|}{ Malestar Emocional } & \multicolumn{2}{|c|}{ Espiritualidad } & \multicolumn{2}{|c|}{$\begin{array}{l}\text { Preconceptos y valoración de las } \\
\text { SPA }\end{array}$} \\
\hline & Protección & Riesgo & Protección & Riesgo & Protección & Riesgo \\
\hline & $\mathrm{N}(\%)$ & $\mathrm{N}(\%)$ & $\mathrm{N}(\%)$ & $\mathrm{N}(\%)$ & $\mathbf{N}(\%)$ & $\mathrm{N}(\%)$ \\
\hline 16 a 20 & $131(57,5)$ & $97(42.5)$ & $181(80,1)$ & $45(19,9)$ & $2(12,0)$ & $162(98,8)$ \\
\hline 21 a 25 & $58(56,9)$ & $44(43,1)$ & $85(78,7)$ & $23(21,3)$ & 0 & $77(100)$ \\
\hline 26 a 30 & $13(72,2)$ & $5(27.8)$ & $13(72,2)$ & $5(27,8)$ & 0 & $14(100)$ \\
\hline 31 a 35 & $1(100)$ & 0 & $1(100)$ & 0 & 0 & $1(100)$ \\
\hline $\mathrm{NR}^{*}$ & $6(60,0)$ & $4(40,0)$ & $8(80,0)$ & $2(20,0)$ & 0 & $8(100)$ \\
\hline \multirow[t]{2}{*}{ Total } & $209(58,2)$ & $150(41.8)$ & $288(79,3)$ & $75(20,7)$ & $2(0,8)$ & $262(99,2)$ \\
\hline & \multicolumn{2}{|c|}{$\begin{array}{l}\text { Habilidades sociales y de } \\
\text { autocontrol }\end{array}$} & \multicolumn{2}{|c|}{$\begin{array}{l}\text { Satisfacción con relaciones } \\
\text { interpersonales }\end{array}$} & \multicolumn{2}{|c|}{$\begin{array}{c}\text { Permisividad social y accesibilidad } \\
\text { a las SPA }\end{array}$} \\
\hline \multirow[t]{2}{*}{ Grupos de edad } & Protección & Riesgo & Protección & Riesgo & Protección & Riesgo \\
\hline & $N(\%)$ & $N(\%)$ & $\mathrm{N}(\%)$ & $\mathrm{N}(\%)$ & $N(\%)$ & $\mathrm{N}(\%)$ \\
\hline 16 a 20 & $81(35,2)$ & $149(64,8)$ & $226(98,3)$ & $4(1,7)$ & $63(28,1)$ & $161(71,9)$ \\
\hline 21 a 25 & $42(38,2)$ & $68(61,8)$ & $106(100)$ & 0 & $33(30,6)$ & $75(69,4)$ \\
\hline 26 a 30 & $9(47,4)$ & $10(52,6)$ & $18(94,7)$ & $1(5,3)$ & $4(22,2)$ & $14(77,8)$ \\
\hline 31 a 35 & $1(100)$ & 0 & $1(100)$ & 0 & 0 & $1(100)$ \\
\hline $\mathrm{NR}^{*}$ & $5(50,0)$ & $5(50,0)$ & $8(80,0)$ & $2(20,0)$ & $5(50,0)$ & $5(50,0)$ \\
\hline Total & $138(37,3)$ & $232(62,7)$ & $359(98,1)$ & $7(1,9)$ & $105(29,1)$ & $256(70,9)$ \\
\hline
\end{tabular}

*NR: No Responde

En la mayoría de los estudiantes que viven con los dos padres, los preconceptos y valoración de las
SPA, permisividad social y accesibilidad a las SPA están presentes como factores de riesgo (Tabla 3).

Tabla 3 - Factores de Riesgo y de protección para el uso de drogas según dependencia económica de los estudiantes

\begin{tabular}{|c|c|c|c|c|c|c|}
\hline \multirow{3}{*}{$\begin{array}{l}\text { Dependencia } \\
\text { económica }\end{array}$} & \multicolumn{2}{|c|}{ Malestar Emocional } & \multicolumn{2}{|c|}{ Espiritualidad } & \multicolumn{2}{|c|}{$\begin{array}{c}\text { Preconceptos y valoración de } \\
\text { las SPA }\end{array}$} \\
\hline & Protección & Riesgo & Protección & Riesgo & Protección & Riesgo \\
\hline & n (\%) & n (\%) & n (\%) & n (\%) & n (\%) & n (\%) \\
\hline Alguno de los padres & $47(56,0)$ & $37(44,0)$ & $70(79,5)$ & $18(20,5)$ & 0 & $64(100)$ \\
\hline Padre y Madre & $139(59,1)$ & 96 (40.9) & $184(78,6)$ & $50(21,4)$ & $2(1,2)$ & $168(98,8)$ \\
\hline Otro & $21(55,3)$ & $17(44.7)$ & $33(84,6)$ & $6(15,4)$ & 0 & $30(100,0)$ \\
\hline $\mathrm{NR}^{*}$ & $2(100)$ & 0 & $1(50,0)$ & $1(50,0)$ & 0 & 0 \\
\hline Total & $209(58,2)$ & $150(41.8)$ & $288(79,3)$ & $75(20,7)$ & $2(0,8)$ & $262(99,2)$ \\
\hline \multirow{3}{*}{$\begin{array}{l}\text { Dependencia } \\
\text { económica }\end{array}$} & \multicolumn{2}{|c|}{$\begin{array}{c}\text { Habilidades sociales y de } \\
\text { autocontrol }\end{array}$} & \multicolumn{2}{|c|}{$\begin{array}{l}\text { Satisfacción con relaciones } \\
\text { interpersonales }\end{array}$} & \multicolumn{2}{|c|}{$\begin{array}{c}\text { Permisividad social y } \\
\text { accesibilidad a las SPA }\end{array}$} \\
\hline & Protección & Riesgo & Protección & Riesgo & Protección & Riesgo \\
\hline & n (\%) & n (\%) & n (\%) & n (\%) & n (\%) & n (\%) \\
\hline Alguno de los padres & $31(34,1)$ & $60(65,9)$ & $89(98,9)$ & $1(1,1)$ & $24(27,7)$ & $65(73,0)$ \\
\hline Padre y Madre & $93(39,2)$ & $144(60,8)$ & $232(98,3)$ & $4(1,7)$ & $68(29,6)$ & $162(70,4)$ \\
\hline Otro & $13(32,5)$ & $27(67,5)$ & $37(97,4)$ & $1(2,6)$ & $11(27,5)$ & $29(72,5)$ \\
\hline $\mathrm{NR}^{*}$ & $1(50,0)$ & $1(50,0)$ & $1(50,0)$ & $1(50,0)$ & $2(100)$ & 0 \\
\hline Total & $138(37,3)$ & $232(62,7)$ & $359(98,1)$ & $7(1,9)$ & $105(29,1)$ & $256(70,9)$ \\
\hline
\end{tabular}

*NR: No Responde

La mayoría de los estudiantes que consumen sustancias licitas como el tabaco y el alcohol está en riesgo en el dominio preconceptos y valoraciones, permisividad social y accesibilidad frente al consumo de las SPA. Estos dominios igualmente son de riesgo para los estudiantes que consumen sustancias ilícitas, principalmente la Marihuana (Tabla 4). 
Tabla 4 - Frecuencia de consumo de substancias por factores protectores y de riesgo

\begin{tabular}{|c|c|c|c|c|c|c|c|}
\hline & & \multicolumn{2}{|c|}{ Malestar Emocional } & \multicolumn{2}{|c|}{ Espiritualidad } & \multicolumn{2}{|c|}{$\begin{array}{c}\text { Preconceptos y valoración de } \\
\text { las SPA }\end{array}$} \\
\hline \multicolumn{2}{|c|}{ Sustancia de consumo } & Protección & Riesgo & Protección & Riesgo & Protección & Riesgo \\
\hline & & $\mathbf{N}(\%)$ & N (\%) & $\mathbf{N}(\%)$ & $\mathbf{N}(\%)$ & $\mathbf{N}(\%)$ & $\mathbf{N}(\%)$ \\
\hline \multirow{2}{*}{ Alcohol } & No & $6(60)$ & $4(40)$ & $6(55)$ & $5(46)$ & $1(14)$ & $6(86)$ \\
\hline & $\mathrm{Si}$ & $202(58)$ & $146(42)$ & $282(80)$ & $69(20)$ & $1(0.4)$ & $256(99.6)$ \\
\hline \multirow{2}{*}{ Tabaco } & No & $85(64)$ & $48(36)$ & $114(84)$ & $21(16)$ & $2(2)$ & $91(98)$ \\
\hline & $\mathrm{Si}$ & $123(55)$ & $102(45)$ & $174(77)$ & $53(23)$ & 0 & $171(100)$ \\
\hline \multirow{2}{*}{ Marihuana } & No & $182(57)$ & $135(43)$ & $261(81)$ & $60(19)$ & $2(1)$ & $226(9.9)$ \\
\hline & $\mathrm{Si}$ & $25(63)$ & $15(38)$ & $26(65)$ & $14(35)$ & 0 & $36(100)$ \\
\hline \multirow{2}{*}{ Opióides } & No & 207 (59) & $146(41)$ & $284(80)$ & $73(20)$ & $2(1)$ & 258 (99) \\
\hline & $\mathrm{Si}$ & 0 & $4(100)$ & $3(75)$ & $1(25)$ & 0 & $4(100)$ \\
\hline \multirow{2}{*}{$\begin{array}{l}\text { Consumo de } \\
\text { alucinógenos }\end{array}$} & No & $197(58)$ & $142(42)$ & $340(98)$ & $6(2)$ & $2(1)$ & $246(99)$ \\
\hline & $\mathrm{Si}$ & $9(64)$ & $5(36)$ & $14(100)$ & 0 & 0 & $13(100)$ \\
\hline & & \multicolumn{2}{|c|}{$\begin{array}{l}\text { Habilidades sociales y de } \\
\text { autocontrol }\end{array}$} & \multicolumn{2}{|c|}{$\begin{array}{l}\text { Satisfacción con relaciones } \\
\text { interpersonales }\end{array}$} & \multicolumn{2}{|c|}{$\begin{array}{l}\text { Permisividad social y } \\
\text { accesibilidad a las SPA }\end{array}$} \\
\hline \multicolumn{2}{|c|}{ Sustancia de consumo } & Protección & Riesgo & Protección & Riesgo & Protección & Riesgo \\
\hline & & $\mathrm{N}(\%)$ & $\mathrm{N}(\%)$ & $\mathrm{N}(\%)$ & $\mathrm{N}(\%)$ & $\mathrm{N}(\%)$ & $\mathrm{N}(\%)$ \\
\hline \multirow{2}{*}{ Alcohol } & No & $5(50)$ & $5(50)$ & $10(91)$ & $1(9,1)$ & $4(36)$ & $7(64)$ \\
\hline & $\mathrm{Si}$ & $132(37)$ & $227(63)$ & $349(99)$ & $5(1.4)$ & $100(29)$ & $249(71)$ \\
\hline \multirow{2}{*}{ Tabaco } & No & $59(43)$ & $78(57)$ & 133 (99) & $2(1.5)$ & $56(42)$ & $77(58)$ \\
\hline & $\mathrm{Si}$ & $78(34)$ & $154(66)$ & $226(98)$ & $4(1.7)$ & $48(21)$ & $179(79)$ \\
\hline \multirow{2}{*}{ Marihuana } & No & $115(35)$ & $213(65)$ & $318(98)$ & $6(1.9)$ & $96(30)$ & $223(70)$ \\
\hline & $\mathrm{Si}$ & $22(55)$ & $18(45)$ & $40(10.0)$ & 0 & $8(20)$ & $32(80)$ \\
\hline \multirow{2}{*}{ Opióides } & No & $136(37)$ & $228(63)$ & 354 (98) & $6(1,7)$ & $102(29)$ & $253(71)$ \\
\hline & $\mathrm{Si}$ & $1(25)$ & $3(75)$ & $4(100)$ & 0 & $2(50)$ & $2(50)$ \\
\hline \multirow{2}{*}{$\begin{array}{l}\text { Consumo de } \\
\text { alucinógenos }\end{array}$} & No & $274(80)$ & $69(20)$ & 99 (29) & $242(71)$ & $129(37)$ & $221(63)$ \\
\hline & $\mathrm{Si}$ & $10(71)$ & $4(29)$ & $4(29)$ & $10(71)$ & $7(50)$ & $7(50)$ \\
\hline
\end{tabular}

Para promover los factores protectores y prevenir los de riesgo es importante conocer la frecuencia de los ítems contemplados en el cuestionario por dominio en el grupo de estudiantes. Se describen la frecuencia de cada uno de ellos en la Tabla 5.

Tabla 5 - Frecuencia de los cinco dominios estudiados

\begin{tabular}{|c|c|c|}
\hline Dominio e ítems & $\begin{array}{c}\text { No } \\
\text { n (\%) }\end{array}$ & $\begin{array}{c}\mathrm{Si} \\
\mathrm{n}(\%)\end{array}$ \\
\hline \multicolumn{3}{|l|}{ Malestar Emocional } \\
\hline Constantemente me siento aburrido & $280(74,9)$ & $94(25,1)$ \\
\hline Me siento muy afectado por mis problemas & $250(66,8)$ & $124(33,2)$ \\
\hline Tengo problemas para dormir o me despierto con facilidad & $313(84,1)$ & $59(15,9)$ \\
\hline Tengo pensamientos repetitivos & $207(55,2)$ & $168(44,8)$ \\
\hline Tengo dificultad para relajarme & $284(75,9)$ & $90(24,1)$ \\
\hline Tengo pensamientos que me fastidian & $235(63,0)$ & $138(37)$ \\
\hline Paso fácilmente de la tristeza a la alegría o al revés & $179(48,6)$ & $189(51,4)$ \\
\hline Me irrito con facilidad & $214(57,5)$ & $158(42,5)$ \\
\hline Me relajo & $54(14,5)$ & $318(85,5)$ \\
\hline Me siento angustiado con frecuencia & $252(67,7)$ & $120(32,3)$ \\
\hline Me tensiono fácilmente & $183(49,2)$ & $189(50,8)$ \\
\hline Me pongo triste fácilmente & $197(53)$ & $175(47)$ \\
\hline \multicolumn{3}{|l|}{ Satisfacción con relaciones interpersonales. } \\
\hline Cuento con alguien para hablar si lo necesito & $15(4,0)$ & $360(96,0)$ \\
\hline Le agrado a otras personas de mi edad & $13(3,5)$ & $361(96,5)$ \\
\hline
\end{tabular}


Tabla 5 - (continuación)

\begin{tabular}{|c|c|c|}
\hline Dominio e ítems & $\begin{array}{c}\text { No } \\
\mathrm{n}(\%)\end{array}$ & $\begin{array}{l}\text { Si } \\
\mathrm{n}(\%)\end{array}$ \\
\hline Me siento satisfecho con mis amistades & $30(8,1)$ & $342(91,9)$ \\
\hline Mis amigos me tienen en cuenta para las actividades que realizan & $16(4,70)$ & $375(95,7)$ \\
\hline Consigo amigos fácilmente & $64(17,20)$ & $308(82,8)$ \\
\hline Disfruto compartiendo actividades de recreación con mis amigos & $4(1,10)$ & $369(98,9)$ \\
\hline Consulto a alguno de mis padres sobre asuntos de mi vida personal & $83(22,3)$ & $289(77,7)$ \\
\hline Mis amigos me ayudan a enfrentar mis problemas & $77(20,6)$ & $296(79,4)$ \\
\hline Hay personas a quienes yo les importo & $6(1,6)$ & $367(98,4)$ \\
\hline \multicolumn{3}{|l|}{ Espiritualidad } \\
\hline Tengo fe en un poder más grande que yo & $92(24,7)$ & 0,753 \\
\hline No soy una persona religiosa & $202(54,7)$ & $167(45,3)$ \\
\hline Me apoyo en la religión cuando tengo problemas & $65(17,4)$ & $309(82,6)$ \\
\hline Creo que existe una fuerza espiritual que puede ayudarme con mis problemas & $45(12,0)$ & $329(88,0)$ \\
\hline Me ayuda rezar o meditar & $97(26,1)$ & $274(73,9)$ \\
\hline He tenido experiencias espirituales o religiosas importantes en mi vida & $82(22,0)$ & $291(78,0)$ \\
\hline \multicolumn{3}{|l|}{ Permisividad social y accesibilidad a las SPA } \\
\hline Los amigos con los que ando piensan que está mal emborracharse & $265(71,0)$ & $108(29,0)$ \\
\hline Los amigos con los que ando piensan que está mal drogarse & $49(13,2)$ & $321(86,8)$ \\
\hline $\begin{array}{l}\text { Siento la necesidad de consumir alcohol o drogas cuando estoy en un bar, discoteca u otro sitio } \\
\text { de ambiente }\end{array}$ & $233(62,8)$ & $138(37,2)$ \\
\hline $\begin{array}{l}\text { Mi primera opción para una salida con mis amigos es un sitio donde pueda tomar o consumir } \\
\text { drogas }\end{array}$ & $310(82,9)$ & $64(17,1)$ \\
\hline Me gusta experimentar nuevas sensaciones & $125(33,5)$ & $248(66,5)$ \\
\hline Tengo algún familiar que bebe hasta emborracharse & $172(46,2)$ & $200(53,8)$ \\
\hline Consumir drogas es normal entre los jóvenes & $170(45,8)$ & $201(54,2)$ \\
\hline Mis amigos incluyen trago, cigarrillo o drogas en las actividades que realizamos & $195(52,3)$ & $178(47,7)$ \\
\hline \multicolumn{3}{|l|}{ Habilidades sociales y de autocontrol } \\
\hline Evito preguntar por temor a hacer el ridículo & $285(76,2)$ & $89(23,8)$ \\
\hline Me siento molesto cuando las cosas no salen a mi manera & $68(18,1)$ & $307(81,9)$ \\
\hline Me preocupo en exceso & $194(51,9)$ & $180(48,1)$ \\
\hline Prefiero ceder para evitar problemas con otras personas & $171(45,6)$ & $204(54,4)$ \\
\hline Me enojo cuando la gente me hace esperar & $71(19,0)$ & $303(81,0)$ \\
\hline Evito herir los sentimientos de alguien a propósito & $32(8,6)$ & $32(8,6)$ \\
\hline Me preocupo cuando rompo una regla & $45(12,1)$ & $328(87,9)$ \\
\hline Pienso mucho antes de actuar & $83(22,3)$ & $289(77,7)$ \\
\hline \multicolumn{3}{|l|}{ Preconceptos y valoración de las SPA } \\
\hline Para las personas de mi edad es difícil conseguir drogas & $32(8,8)$ & $332(91,2)$ \\
\hline Para las personas de mi edad es costoso consumir drogas frecuentemente & $118(32,7)$ & $243(67,3)$ \\
\hline Cuando se bebe en exceso se hace el ridículo & $250(67,0)$ & $123(33,0)$ \\
\hline Las drogas son un problema más grave que el alcohol o el cigarrillo & $337(90,3)$ & $36(9,7)$ \\
\hline
\end{tabular}

\section{Discusión}

En este estudio fue frecuente encontrar como factores de riesgo los dominios "preconceptos y valoración de las SPA", "permisividad social y accesibilidad a las SPA" y "habilidades sociales y autocontrol" en los estudiantes que cursan los primeros semestres. Los hallazgos son concordantes con la literatura, la cual explica que estos comportamientos y actitudes de riesgo se deben a que los estudiantes empiezan procesos para adaptarse al ámbito universitario y a las nuevas presiones académicas y grupales. En esta etapa, los adolescentes están en transición niñez-juventud-adultez y la forma como asimilan los cambios fisiológicos, psicológicos y sociales determinarán el logro de un mayor nivel de independencia y autonomía anhelando mayores cuotas de libertad y a cuestionar los valores adquiridos en la familia, pretendiendo encontrar un sentido de vida propio basado en el conocimiento de sí mismo, de sus gustos, actitudes, limitaciones y preferencias ${ }^{(10,18)}$. La mayor 0 menor adquisición de estas características (personales, sociales, ambientales, familiares, psicológicas) conjuran como factores de riesgo o de protección, facilitando o no la probabilidad de inicio y desarrollo del consumo de drogas manteniendo el sujeto alejado de esta conducta(12,19). No se puede olvidar que los jóvenes sienten la necesidad 
de experimentar sensaciones nuevas e intensas, de estimulaciones fuertes, de conocerse y conocer las propias limitaciones son características muy asociadas a la adolescencia y aspectos frecuentemente mencionados por estos a la hora de argumentar el consumo(20-21), aspectos considerados en el dominio de habilidades sociales y autocontrol para el presente estudio y que mostraron como resultado ser un factor de riesgo.

Al revisar la literatura, los factores de riesgo sociales evidencian que la disponibilidad de las sustancias y la influencia ejercida por los medios de comunicación son los que se sitúan en un lugar preponderante a la hora de determinar la conducta de consumo. Ingerir una sustancia, como todo comportamiento, se produce $y$ toma sentido en un determinado contexto social, en el nuestro se manifiesta la presencia de alcohol, tabaco en lugares cercanos y accesibles y la incorporación de éstos en nuestros estilos de vida (celebraciones, actos sociales, etc. $)^{(16,21-22)}$. La integración cultural de sustancias como el alcohol y la permisividad y aceptación social ejercida a través de actitudes y normas también explican un mayor uso de las mismas ${ }^{(16,21,23)}$. Estos comportamientos sociales inducen a los jóvenes a percibir que las SPA son accesibles y permitidas en su comportamiento no dimensionarlas como riesgo, específicamente para el presente estudio se ven reflejadas en el dominio permisividad social y accesibilidad a las SPA, el cual fue más común como riesgo. Igualmente, la presentación desde los medios de comunicación de personas atractivas, relevantes y próximas a los jóvenes, con los cuales se identifican presentando modelos de conducta de consumo, nublando al sujeto y no permitiéndole ser consciente y capaz de percibir los riesgos de una conducta riesgosa que por ser practicada por un amplio número de personas, cuenta con una aceptación social, mantenida muchas veces sobre informaciones erróneas y falsamente publicitadas ${ }^{(16,21,23)}$. Se ha demostrado que la fácil accesibilidad a la droga es un factor de riesgo en el inicio y mantenimiento del SPA y cuanta más alta la permisividad entendida como los valores y actitudes sociales favorables al consumo(5,21,24).

La percepción que el sujeto tenga de riesgo sobre el consumo de una sustancia es importante, hasta tal punto que diversos estudios han identificado un descenso del nivel de consumo de las sustancias que son percibidas como de mayor riesgo ${ }^{(24-25)}$. Sin embargo, este no parece ser el escenario de la mayoría de los jóvenes del estudio, porque según el dominio preconceptos y valoración no perciben las SPA como riesgo, especialmente las sustancias licitas, lo que invita a intervenir en esta población tratando de concientizarlos del riesgo que implica el consumo de las ilícitas como de las licitas.

La literatura señala que estados de estrés, ansiedad, depresión y/o la necesidad de asumir cambios podrían desencadenar un acercamiento al consumo de drogas, asumiendo falazmente que los efectos farmacológicos de la misma reducirán y/o aliviarán estos estados negativos.

Las personas carentes de recursos personales o psicosociales para hacerle frente y superarlos de otra forma tienen más probabilidad de consumir drogas ${ }^{(21-}$ 22). Estas pueden ser algunas de las razones que los estudiantes podrían tener para estar en riesgo ante el consumo de SPA y específicamente en el dominio malestar emocional, aunque para este estudio la frecuencia de este componente como factor de riesgo no fue tan frecuente es relevante considerarlo para posibles intervenciones de prevención en esta población.

La mayoría de los estudiantes encuestados tienen como factor protector el dominio satisfacción con las relaciones interpersonales. Algunos autores reportan este factor como uno de los más importantes porque consideran que afrontar las relaciones con los otros, ser capaz de interaccionar socialmente expresando sus opiniones y sentimientos les permite a los jóvenes establecer relaciones adecuadas y adaptadas a la realidad, fortaleciendo su personalidad, brindando seguridad y convirtiéndose en una exposición protectora frente al consumo de SPA ${ }^{(5,16)}$. Sin embargo una persona con dificultades para expresar sus opiniones o para desenvolverse adecuadamente en su entorno será más vulnerable a la influencia del grupo. De esta forma, la persona con dificultades en las habilidades sociales, con poca asertividad, difícilmente podrá resistirse a la presión grupal, dejándose involucrar fácilmente en el consumo de SPA, percibiendo erróneamente que su comportamiento generará aceptación en el entorno en el que se desarrolla e igualmente creyendo que los efectos farmacológicos de SPA hacen que se desenvuelva sin limitaciones antes sus pares(19-20); si se adiciona a este escenario la permisividad social y accesibilidad de las SPA, las habilidades sociales y de autocontrol como factores de riesgo no se espera un pronóstico favorable para el consumo de las SPA.

El presente estudio encontró como factores protectores la satisfacción con relaciones interpersonales, espiritualidad y malestar emocional. Esto puede estar relacionado con la teoría del desarrollo social propuesta por Hawkins que describe tres factores de protección que controlan el desarrollo de los comportamientos 
antisociales: los lazos sociales (adhesión y compromiso con la familia, la escuela y los compañeros); las coacciones externas (normas claras y consistentes contra el consumo de drogas mantenidas por personas ligadas al individuo); y las habilidades sociales (poseer estrategias de solución de problemas para afrontar asertivamente las situaciones y resistir las presiones a la transgresión de normas)(21-22). Igualmente un buen nivel de autoestima, un adecuado autocontrol emocional, la cohesión y comunicación familiar, el apego a un grupo de referencia positivo favorecerían en el sujeto frente a conductas desviadas ${ }^{(20,23,25)}$. Esta teoría se encuentra relacionada con la investigación realizada, donde se encuentra que para los estudiantes los amigos, la familia y un ser superior son importantes en su proceso de vida. Parece que la religiosidad, la creencia en el orden moral, el grado de satisfacción personal respecto a la vida actúan como factores de protección ${ }^{(25)}$. La consistencia, responsabilidad y seguridad en las relaciones familiares facilitan el desarrollo de individuos sanos dentro del grupo, brindándoles estabilidad, previsibilidad en las reacciones y consecuencias de diferentes comportamientos y situaciones. Un buen funcionamiento familiar en términos de buena comunicación, implicación, dedicación, afectos y cercanía de los padres correlacionan positivamente con una menor implicación de conducta problema por parte del adolescente(20-21). Los estudiantes de enfermería conviven con los dos padres en el $73,8 \%$ de los casos, con la mamá el $16,9 \%$ y con el padre el 6,2\%, fortaleciendo los lazos de interacción familiar, convirtiéndose en unos de los dominios más destacados como protectores en este estudio.

Para finalizar se debe tener en cuenta que los factores de riesgo pueden estar presentes o no en un caso concreto; cuando un factor de riesgo está presente, es más probable que la persona use o abuse de las drogas, que cuando no lo está. La presencia de un sólo factor de riesgo no es garantía de que se vaya a producir el abuso de drogas y por el contrario la ausencia del mismo no garantiza que el abuso no se produzca, lo mismo sucede en el caso de los factores de protección. El abuso de drogas suele ser impredecible y en todo caso es el resultado de la intervención conjunta de muchos factores ${ }^{(12,21)}$. Por otro lado, aspectos como el nivel cultural, el rendimiento académico, la implicación con el medio escolar y los refuerzos obtenidos por parte de padres y profesores determinan en mayor medida las características diferenciales de los adolescentes no consumidores frente a los consumidores ${ }^{(10,21)}$.

\section{Conclusiones}

Con estos resultados se puede presumir que los estudiantes no dimensionan los riesgos frente al consumo de alcohol y tabaco, lo que los lleva a considerar su consumo como normal y de aceptación social, lo que justifica la importancia de identificar alteraciones emocionales (depresión, ansiedad) en los adolescentes y jóvenes, a fin de dar soporte profesional desde salud mental (psiquiatría, psicología, enfermería) y conformar redes de apoyo frente a los factores de riesgo y los factores de protección. Siendo así, la enfermería, en el acto de cuidado por la vida, debe participar en forma dinámica en la educación de las poblaciones, en la prevención de conductas de riesgo y en el fortalecimiento de factores protectores. De esta forma, es importante fortalecer el currículo de enfermería como eje transversal en la prevención de drogas, desde una perspectiva integral en el cuidado de la calidad de vida de las comunidades. Es también fundamental promover la sensibilización de la comunidad académica para asumir, desde la enfermería, la educación y el cuidado del ser humano, en la prevención de prácticas de riesgo en el consumo de drogas, fortaleciendo estrategias para el trabajo integrado con las comunidades.

\section{Agradecimientos}

Agradecemos a la Comisión Interamericana para el Control del Abuso de Drogas/CICAD de la Secretaria de Seguridad Multidimensional/SSM de la Organización de los Estados Americanos/OEA, la Secretaria Nacional de Políticas sobre Drogas/SENAD do Gabinete de Seguridad Institucional/Brasil, la Escuela de Enfermería de Ribeirao Preto de la Universidad de Sao Paulo y Centro Colaborador de la Organización Mundial de la Salud para el Desarrollo de la Investigación en Enfermería, la población representada en los estudios de investigación, bien como a las autoridades de las universidades representadas por los participantes del Programa En-Line de Especialización en Investigación sobre el Fenómeno de las Drogas, periodos 2006, 2007, 2008 y 2009.

\section{Referencias}

1. República de Colombia. Política Nacional para la Reducción del Consumo de Sustancias Psicoactivas y su impacto. Bogotá. D.C.: Ministerios de la Protección Social; Abril 2007. p. 11-28. Resumen Ejecutivo. 
2. Godue C. La salud en los procesos de globalización y de internacionalización. En: Pontificia Universidad Javeriana. Relaciones internacionales, política social y salud: desafíos en la era de la globalización. Memorias del Foro Internacional. Bogotá: Pontificia Universidad Javeriana; 1997.

3. Comisión Interamericana para el Control del Abuso de Drogas (CICAD). Organización de los Estados Americanos. Evaluación del progreso del Control de Drogas .Informe Hemisférico. Washington, D.C; 20012002. $18 \mathrm{p}$.

4. López JJ, Millar MV. Criterios Diagnósticos. DSM IV TR. Breviario. España: Masson; 2004. 327 p.

5. República de Colombia. Estudio Nacional de Salud Mental Colombia. Fundación FES Social. Cali (CO: Ministerio de la Protección Social; Diciembre 2005. p. 36-40.

6. Martínez-Mantilla JA, Amaya-Naranjo W, Campillo Horacio A, Rueda-Jaimes GE, Campo-Arias Ad, DíazMartínez LA. Consumo de Sustancias psicoactivas en Adolescentes, Bucaramanga, Colombia, 1996-2004. Rev. Salud Pública. 2007;9(2):215-29.

7. Universidad San Buenaventura, Fundación Hogares Claret (CO). Diplomado en consejería de Drogodependencias. Núcleo temático 2. La Formación Técnica y Terapéutica. Medellín. Colombia; 2001. (45):46-51.

8. Wright MGM. La Contribución de la Enfermería frente al fenómeno de las drogas y la violencia en América Latina: Un proceso en construcción. Cienc Enferm. Rev Iberoam Invest. 2002;8(2):9-19.

9. República de Colombia. Política Nacional para la Reducción del Consumo de Sustancias Psicoactivas y su impacto. Bogotá. Ministerio de la Protección Social; Abril 2007. 3 p. Resumen ejecutivo.

10. Moral Jimenez $M$ de la V, Rodriguez Diaz FJ, Sirvent Ruiz C. Factores relacionados con las actitudes juveniles hacia el consumo de alcohol y otras sustancias psicoactivas. Psicothema. 2006;18(1):52-8.

11. Rueda Silva LVE, Malbergier A, Stempliuk VA, Andrade AG. Factors associated with drug and alcohol use among university students. Rev Saúde Pública 2006;40(2):280-8.

12. Osorio Rebolledo EA, Ortega de Medina NM, Pillon SC. Factores de Riesgo Asociados al Uso de Drogas en Estudiantes Adolescentes1. Rev. Latino-Am. Enfermagem. março-abril. 2004;12(nº esp):369-75.

13. Comisión Interamericana para el Control del Abuso de Drogas (CICAD). Organización de los Estados Americanos. Evaluación del progreso del control de drogas. Washington, D.C; 2003. Informe 1-11 Informe Hemisférico.

14. Martínez A. Familia y consumo de drogas desde el Modelo Circumplejo de evaluación familiar. En: Vielva I; Pantoja L; Abeijón JA. Las familias y sus adolescentes ante las drogas. El funcionamiento de la familia con hijos de comportamiento no problemático, consumidores y no consumidores de drogas. Avances Drogodependencias 2001; 11:234-6.

15. Salazar IC, Varela MT, Cáceres DE, Tovar JR. Cuestionario de Factores Riesgo y de protección para el consumo de drogas en jóvenes (FRP-SPA). Grupo de Investigación Psicología, Salud y Calidad de Vida. Pontificia Universidad Javeriana Cali, Colombia. Acta Colombiana Psicol. 2006;9(2):19-30.

16. Salazar IC, Varela MT, Cáceres DEY, Tovar JR. Consumo de drogas con jóvenes Universitarios $y$ su relación de Riesgo y Protección con los Factores psicosociales. Grupo de Investigación Psicología, Salud y Calidad de Vida. Pontificia Universidad Javeriana Cali, Colombia. Univ. Psychol. Bogotá. Octub-Dic 2006; 5(3):521-34.

17. Ley 266 de 25 enero 1996 (CO). Tribunal Nacional Ético de Enfermería. Por la cual se reglamenta la profesión de enfermería en Colombia y se dictan otras disposiciones.

Diario Oficial n. 42.710.

18. Corredor Cecilia. República de Colombia. Ministerio de Justicia y del Derecho. Dirección Nacional de Estupefacientes. Unidad Administrativa Especial Entidad de Coordinación Nacional. Sistema de Vigilancia Epidemiológica para Sustancias Psicoactivas VESPA. Colombia; 2000. 11 p.

19. Trujillo ÁM, Santacana MF, Pérez Gómez A. Uso de sustancias y percepción de riesgo: Estudio comparativo entre jóvenes de Bogotá y Barcelona. Adicciones. 2007;19(2):179-90.

20. Pons Diez J, Berjano Peirats E. El consumo abusivo de alcohol en la adolescencia. Un modelo explicativo desde la psicología social. Plan Nacional de Drogas. Madrid; 1999.

21. Laespada MT. Factores de Riesgo y de Protección Frente al Consumo de Drogas: Hacia un Modelo Explicativo del Consumo de Drogas en Jóvenes de la CAPV. España; Julio 2004. 134 p.

22. Elzo J, Elorza MA, Laespada MT. Alcoholismo juvenil. Reflexiones y sugerencias de actuación ante una realidad contrastada. Bilbao: Universidad de Deusto, Instituto Deusto de Drogodependencias; 1994. p. 55-78. 
23. Flay BR, Petraitis J. Aspectos metodológicos en la investigación de medidas preventivas del consumo de drogas: fundamentos teóricos. En: Leukefeld CG, Bukoski WJ, editors. Estudios sobre intervenciones en la prevención del abuso de drogas: aspectos metodológicos. Centro de Estudios de Promoción de la Salud. Madrid; 1995. p. 83-108.

24. Johnston LD, Leukefeld CG, Bukoski WJ. Contribuciones de la epidemiología de las drogas al campo de la prevención del abuso de drogas. Estudios sobre intervenciones en la prevención del abuso de drogas: aspectos metodológicos. Centro de Estudios de Promoción de la Salud. Madrid; 1995. p. 83-108.

25. Luengo MA, Romero Tamames E. La Prevención del Consumo de Drogas y la Conducta Antisocial en la Escuela. Análisis y Evaluación de un programa. Santiago: Universidad de Santiago de Compostela; 1998: p. 88-90. 